\title{
Special Session 2: \\ Cosmic Evolution of Groups and Clusters
}

\author{
J. M. Vrtilek ${ }^{1}$ and L. P. David ${ }^{2}$ \\ ${ }^{1}$ Harvard-Smithsonian Center for Astrophysics, Cambridge, MA 02138, USA \\ email: jvrtilek@cfa.harvard.edu \\ ${ }^{2}$ Harvard-Smithsonian Center for Astrophysics, Cambridge, MA 02138, USA, \\ email: Idavid@cfa.harvard.edu
}

Session chairs and section authors:

Yipeng Jing (Shanghai Astronomical Observatory)

Christine Jones (Center for Astrophysics)

Elke Roediger (Hamburg Observatory)

Sebastian Heinz (University of Wisconsin)

Jeremy Lim (Hong Kong University)

Daisuke Nagai (Yale University)

Jan Vrtilek (Center for Astrophysics)

Larry David (Center for Astrophysics)

Diana Worrall (University of Bristol)

Paulo Lopes (Universidade Federal do Rio de Janeiro)

Matthew Colless (Australian Astronomical Observatory)

Simon Lilly (ETH Zürich)

\begin{abstract}
During the past decade observations across the electromagnetic spectrum have led to broad progress in the understanding of galaxy clusters and their far more abundant smaller siblings, groups. From the X-rays, where Chandra and $X M M$ have illuminated old phenomena such as cooling cores and discovered new ones such as shocks, cold fronts, bubbles and cavities, through rich collections of optical data (including vast and growing arrays of redshifts), to the imaging of AGN outbursts of various ages through radio observations, our access to cluster and group measurements has leaped forward, while parallel advances in theory and modeling have kept pace.

This Special Session offered a survey of progress to this point, an assessment of outstanding problems, and a multiwavelength overview of the uses of the next generation of observatories. Holding the symposium in conjuction with the XXVIIIth General Assembly provided the significant advantage of involving not only a specialist audience, but also interacting with a broad cross-section of the world astronomical community.
\end{abstract}

Keywords. black hole physics, galaxies: clusters: general, galaxies: cooling flows, galaxies: evolution, galaxies: intergalactic medium, galaxies: jets

\section{Preface}

In the following pages represent an attempt to summarize the presentations and discussion at Special Session 2 held at the XXVIIth IAU General Assembly. The intent, where possible, has been to emphasize discussion of the next generation of problems on which each area of investigation might profitably focus in the near future.

The twelve sessions, spread across five days in the first week of the General Assembly, focused in turn on the four major topics of cosmology and cluster formation, cooling flows 
and AGN feedback, the non-thermal properties of clusters, and the environmental impact of galaxy evolution in clusters. The Special Session offered 74 invited and contributed talks and over 60 posters. We particularly wish to express appreciation to the many presenters of talks and posters from whose research, contributions, and discussion this meeting was built.

We thank the session chairs whose contributions included the preparation of the session remarks presented below, and the Local Organizing Committee and the IAU management, who made the entire meeting possible.

We especially acknowledge the SpS2 organizing committee, without whose work this session would have been impossible. They are:

Monique Arnaud (Commissariat a l'energie atomique/Saclay, France)

Paulo Lopes (Universidade Federal do Rio de Janeiro, Brazil)

D J Saikia (National Centre for Radio Astrophysics, India)

Omar Lopez-Cruz (INAOE, Mexico)

Eugene Churazov (Space Research Institute (IKI), Russia)

Sabine Schindler (University of Innsbruck, Austria)

Diana Worrall (University of Bristol, UK)

Matthew Colless (Australian Astronomical Observatory, Australia)

Manolis Plionis (National Observatory of Athens, Greece)

Yipeng Jing (Shanghai Astronomical Observatory, China)

Jeremy Lim (Hong Kong University, China)

Laurence David (Center for Astrophysics, USA)

Jan Vrtilek (Center for Astrophysics, USA)

\section{Cosmology: SZ Studies}

\section{Session chair and section author: Yipeng Jing}

We highlight the recent results presented in the "Cosmology-SZ studies" session on cosmological applications of the Sunyaev-Zel'dovich (SZ) observations of clusters of galaxies. The South Pole Telescope Sunyaev-Zel'dovich Cluster Survey has led to several major cosmological results on dark energy equation of state, neutrino mass, and number of neutrino species. Observations with MUSTANG on the Green Bank Telescope have resulted in the highest resolution SZ effect images to date, and are revealing complex pressure substructures in intermediate redshift clusters. Future observations with ALMA, with Planck, and with the next generation of instruments on SPT will reveal much better complicated cluster thermodynamics, provide important clues to gas processes in clusters, and put more stringent constraints on cosmological parameters.

The session began with an introductory talk by Mark Birkinshaw on the SunyaevZel'dovich (SZ) effect and on the cluster and group surveys using this effect. The SZ observations can provide an almost mass-limited and distance-independent measure of the gas content of a cluster of galaxies. Recent surveys have found many clusters using the effect. The observations are powerful probes to cosmological models and to formation and physical processes of clusters of galaxies. His talk focused on the significant differences between typical clusters found through the Sunyaev-Zeld́ovich effect, X-ray emission, and galaxy content, and discussed how recent imaging in the X-ray and SZ effects illustrates the origins of some of the these differences and indicates the underlying physical processes. Other speakers have presented recent surveys and new results from these surveys. 
Bradford Benson presented recent results based on the South Pole Telescope SunyaevZel'dovich Cluster Survey. The South Pole Telescope is a 10-meter telescope optimized for sensitive, high-resolution measurements of cosmic microwave background (CMB) anisotropy and of the millimeter-wavelength sky. In November 2011, the SPT completed the 2500 deg$^{2}$ SPT-SZ survey. The survey has led to several major cosmological results, derived from measurements of the fine angular scale primary and secondary CMB anisotropies, the discovery of galaxy clusters via the Sunyaev-Zel'dovich (SZ) effect and the resulting mass-limited cluster catalog, and the discovery of a population of distant, dusty star forming galaxies (DSFGs). Notably, the SPT-SZ survey has led to the best current measurement of the primordial fine-scale CMB anisotropy power spectrum, and recently released a catalog of 158 SZ-selected optically-confirmed clusters from the first $720 \mathrm{deg}^{2}$ of the survey, more than doubling the number of comparably massive clusters discovered at redshift $z>0.5$. The SPT-SZ data have been used to demonstrate significant improvements in the constraints on the dark energy equation of state, $w$, and the sum of the neutrino masses, $\Sigma m_{\nu}$, measuring $w=-0.973 \pm 0.063$ and $\Sigma m_{\nu}<0.28 \mathrm{eV}$ at $95 \%$ confidence, a factor of 1.25 and 1.4 improvement, respectively, over the constraints without SPT-SZ cluster data. Adding the number of relativistic particle species as a free parameter, the SPT-SZ data has been used to measure $N_{\text {eff }}=3.91 \pm 0.42$ and constrain $\Sigma m_{\nu}<0.63 \mathrm{eV}$ at $95 \%$ confidence, intriguingly $2 \sigma$ higher than the value of 3.046 expected from only three neutrinos and early energy injection from electron-positron annihilation at the end of neutrino freeze-out. The full SPT-SZ $2500 \mathrm{deg}^{2}$ catalog is expected to be released in 2013, which consists of $\sim 400$ SZ-selected clusters, $\sim 80 \%$ of them new discoveries. In combination with multi-wavelength X-ray and weak lensing observations, he expected the combined CMB power spectrum and SPT-SZ cluster data sets to constrain $\delta(w) / w=5 \%$, a constraint on dark energy from the growth of structure comparable to current geometric-based constraints, a powerful systematic test of our standard dark energy paradigm.

Suzanne Staggs presented results based on the Atacama Cosmology Telescope (ACT) observations. The Atacama Cosmology Telescope (ACT) is a 6-m special purpose telescope designed to measure the CMB. Its first receiver had channels at $148 \mathrm{GHz}, 218 \mathrm{GHz}$ and $277 \mathrm{GHz}$. ACT observes from a site at $5300 \mathrm{~m}$ in the Atacama Desert in Chile. This midlatitude site allows ACT to map regions of the sky in which there exist substantial data from surveys at other wavelengths. The talk focued on ACT's measurements of clusters and groups via their Sunyaev-Zeldovich effect, through direct blind detection as well as statistical analyses involving stacking, measurement of the 3-point function in the maps, and the impact on the angular power spectrum.

Tony Mroczkowski presented recent high angular resolution (9") Sunyaev-Zel'dovich effect (SZE) observations with MUSTANG, a 90-GHz bolometric receiver on the 100meter Green Bank Telescope (GBT). MUSTANG is currently imaging a sample of clusters with complementary Chandra X-ray observations, HST optical observations that probe the mass distribution through strong and weak lensing, radio observations that probe the non-thermal component of the intracluster gas, and lower resolution SZE observations that can recover larger scales $\left(>1^{\prime}\right)$. The MUSTANG observations, which are used to assess the impact of substructure on SZE scaling relations, are among the highest resolution SZE images to date, and are revealing complex pressure substructures in intermediate redshift clusters. Combined, these observations reveal complicated cluster thermodynamics, which must be understood in order to use clusters as cosmological probes. 
Ruediger Kneissl reported the status of the Sunyaev-Zeldovich observations of clusters with the Atacama Large Millimeter/submillimeter Array (ALMA). The radio array, with an array of $5012 \mathrm{~m}$ antennas, the Atacama Compact Array of $127 \mathrm{~m}$ antennas and 4 single dish $12 \mathrm{~m}$ antennas, will offer high sensitivity and high angular resolution for SZ observations of galaxy clusters at frequencies below and above the peak of the cosmic microwave background spectrum, i.e., as decrements and increments. The interferometric technique, the large range of angular scales and the wide frequency coverage should provide high quality, direct images of the small scale gas pressure distribution, and at the same time allow for the rejection of confusing signals, in particular the emission from compact sources. He presented the test data taken during commissioning activities, and outlined future opportunities for science observations with ALMA. He emphasized that the observations will be very powerful for observing substructures in the clusters of galaxies.

Bradford Benson has briefly outlined future plans of the SPT. In 2012, the SPT was equipped with a new polarization sensitive camera, SPTpol, that will detect the contribution to the CMB polarization power spectrum from lensing by large scale structure (the so-called "lensing $B$-modes") and, on larger angular scales, detect, or set an improved upper limit on, the primordial inflationary signal ("gravitational-wave $B$-modes"), thereby constraining the energy scale of inflation. The SPTpol survey will survey about onequarter the area, three times as deep as SPT-SZ, and expects to discover an equal number of clusters, typically finding higher-redshift, lower-mass clusters. Development is underway for SPT-3G, the third-generation camera for SPT. In 2016, they will begin the SPT-3G survey, which we expect to find a factor of $\sim 20$ more clusters than SPT-SZ and be sensitive enough to calibrate cluster masses to $\sim 3 \%$ from CMB cluster lensing.

Jose Diego, on behalf of Planck Collaboration, present some of the latest results of Planck on galaxy clusters through the Sunyaev-Zel'dovich effect. This talk described some new results found combining Planck SZ data with X-ray data on galaxy clusters.

Melanie Johnston-Hollit described the growing importance of lower-frequency radio observations in the study of clusters, with emphasis on relics, halos, and the role of AGN, with overarching goals including the understanding of the generation of magnetic fields, the origin of cosmic rays, and the effects of merger bias on cluster cosmology. The diffuse nature of the sources makes observations particularly challenging. Systems of future interest include the Australia SKA pathfinder and the Murchison Wide Field Array.

\section{Cosmology: X-rays and high-z clusters}

\section{Session chair: Christine Jones}

This session focused on the role of X-ray observations and the study of high-z clusters in modern cosmology. It included the following talks:

Steven Allen on "Cosmology using clusters of galaxies";

Rene Fassbender on "The X-ray luminous galaxy cluster population at $0.9<z \lesssim 1.6$ "; Masayuki Tanaka on "Quiescent early-type galaxies in $z>1.5$ groups";

Yuying Zhang on "Probing substructures in galaxy clusters and resulting systematic in cosmological applications with X-ray and optical surveys"; and

Joana Santos on "Deep Chandra observation of the galaxy cluster WARPJ1415.1+3612 at $\mathrm{z}=1$ : an evolved cool- core cluster at high redshift". 


\title{
4. Large scale properties of clusters and cluster merging
}

\author{
Session chair and section author: Elke Roediger
}

The past decade has made it clear that clusters of galaxies are not relaxed objects. Many clusters show substructure of various kinds and merger or AGN activity. Despite observational challenges, deep observations of cluster outskirts have made the regions of ongoing cluster growth accessible.

\section{Large-scale structure}

Studying the large-scale properties of clusters requires observing their outskirts. This is challenging, because both the galaxy density as well as the ICM density are low. Consequently, the ICM emission is faint, and decreases below $30 \%$ of the background level. Moreover, large field of views (FOVs) are required to cover a significant fraction of the outskirts of nearby clusters. Reliable spectroscopic measurements in cluster outskirts require careful background modelling. Miller et al. (2012) combine the virtues of different X-ray observatories, i.e. the low and stable background of Suzaku, the sensitivity of XMM-Newton and the high spatial resolution of Chandra. These and other work (Akamatsu et al. 2011, Walker et al. 2012) find declining temperature profiles beyond $\sim 0.2 r_{200}$, in broad agreement with theoretical predictions from cosmological simulations (e.g., Burns et al. 2010). The entropy profiles appear to flatten around $0.5 r_{200}$ and even decline beyond $\sim 0.7 r_{200}$ in some cases (Walker et al. 2012). Furthermore, the clusters show an increased degree of azimuthal asymmetry in their outskirts, because accretion takes place preferentially along the large-scale filaments (see also Eckert et al. 2012). In addition to the faint ICM emission, the unknown nature of the ICM at large radii complicates the interpretation of the data. For example, gas clumping can lead to an over-estimate of the ICM density and an under-estimate of the entropy. Thus, some observations suggest declining entropy profiles in the very outskirts and even apparent baryon fractions above the cosmic average (e.g., in Perseus, Simionescu et al. 2011). Other observations agree well with theoretical predictions (e.g. RXCJ 0605, Miller et al. 2012) and show increasing entropy profiles out to the last data point. Recent work also started taking into account the baryons trapped in stars inside galaxies (Lin et al. 2012). While the stellar mass content does not seem to have evolved since redshift 0.6, the ICM fraction has increased in agreement with the self-similar model.

\section{Mergers}

Understanding cluster mergers, their signatures and their effects on clusters is essential if we want to use clusters as cosmological probes. Multiple approaches are currently made, both observationally and theoretically. Mergers are known to leave traces in the ICM that are detected in X-ray observations. Textbook examples include the bullet cluster (Markevitch et al. 2002) and Abell 3667 (Vikhlinin \& Markevitch 2002). Mergers must lead to bulk motions and turbulence in the ICM. The direct observation of radial motions in the ICM requires, however, high spectral resolution. The current X-ray observatories provide only upper limits on turbulence (Sanders et al. 2010). Bulk motions have been measured for the merging cluster Abell 2256, where the radial velocity of the main core and the subcore could be separated (Tamura et al. 2011, 2012). While the relative velocity of $1500 \mathrm{~km} / \mathrm{s}$ between both subclusters is large, measurements of the upcoming ASTRO-H observatory will be significantly more sensitive (Shang \& Oh 2012). The radio 
band has provided the link between mergers and the acceleration of relativistic particles by the observation of radio halos and relics (e.g., van Weeren et al. 2010). These observations will give insights in the physics of particle acceleration mechanisms, turbulence and shocks in the dilute ICM plasma as well. Additionally, mergers can be traced in the galactic or stellar components. Tidal stripping of stars from galaxies leads to a faint, but ubiquitous intra-cluster light, including plumes, shells and streams around cluster galaxies (e.g., Mihos et al. 2005; Janowiecki et al. 2010 for Virgo galaxies). Arnaboldi et al. (2012) present a recent study on the diffuse light distribution in the center of the Hydra I cluster. An off-center envelope and two tidal streams are found in both the diffuse light and in the kinematic data, which enables us to witness the tidal disruption of two companion galaxies. Cluster mergers may even indirectly influence the evolution of their galaxies due to the related shocks. Owers et al. (2012) find "jellyfish" galaxies in Abell 2744. Chandra observations reveal the ICM in Abell 2744 to be disturbed by an ongoing merger. The member galaxies of this cluster separate into two kinematically and spatially distinct subgroups. The "jellyfish" galaxies are star forming galaxies with blue, star forming tails with knots and filaments. Three of these galaxies are found in the region where the ICM must have been shocked recently by the merger, and the increase in ICM pressure may have caused an increased star formation in these galaxies and their tails. However, such a shock-triggered star formation enhancement seems to be a rare phenomenon, since only 4 jellyfish galaxies known so far.

\title{
$A G N$
}

The impact of AGN on the cluster cores has been discussed extensively in the recent decade (e.g., review by McNamara \& Nulsen 2007). However, AGN outbursts can reach cluster-wide scales, e.g. in Hydra A (Nulsen et al. 2005) or MS0735 (McNamara et al. 2009). Now that observations can measure the ICM properties well beyond the cluster core, we can truly start to use the ICM as a calorimeter. Chaudhuri et al. (2012) successfully compare a simple model of energy deposition into the ICM, which consists of pre-heating as well as central AGN heating, to observational data.

\section{Cluster simulations and theory: Computational Modeling of Clusters}

\author{
Session chair and section author: Sebastian Heinz
}

Cluster cosmology has entered an era where precision matches that of other cosmological probes, and where accurate theoretical understanding of observational signatures of clusters is critical for cluster cosmology to advance. Computational models are integral for this next step, allowing a detailed understanding of non-linear physics that will affect $\mathrm{X}$-ray and SZ signatures of clusters. We will highlight some of the recent advances and key next steps in this field.

\section{Cluster Dynamics: Cluster Outskirts}

The natural bias of X-ray observations to observe the cluster core (field of view and, more critically, emission measure) has focused much of the attention in cluster studies on the inner $100 \mathrm{kpc}$ or so. The session on feedback addressed this important region. However, the advent of SZ observations has focused attention outward toward scales 
approaching the Virial radius. Understanding cluster density and temperature profiles out to large radii is therefore critical, both observationally and theoretically.

Observationally, Suzaku has provided the necessary sensitivity to allow such studies Simionescu et al. (2011). A key result from the observations of Perseus are a clear increase in the Baryon fraction with cluster radius, exceeding the cosmic mean. Clearly, this effect has to be understood for proper mass models of clusters from X-ray data to be derived.

The natural interpretation of this effect has been that the medium in the outskirts of clusters is clumpy (leading to a bias in the gas density estimate). Additional evidence for a clumpy gas distribution was presented for the cluster A133 from Chandra data by Vikhlinin et al. (in prep), as well as from a stacking analysis of Rosat data, which also show an enhanced baryon fraction (Eckert et al. 2012).

Simulations have, in fact, predicted clumping Nagai \& Lau (2011). Understanding the extent to which clusters are thus out of equilibrium at large scales and what the effect of clumping is on other cluster observables is one of the key next steps in cluster modeling.

Given that turbulence and clumping may well happen on small scales, it will be important to benchmark numerical results from different codes against each other. In a sense, this question is similar to the study of "cold flows", where mass resolution as well as the proper spatial resolution of dynamical instabilities are required, requirements for which the capabilities of SPH and grid codes classically diverge. It will thus be interesting to see how moving mesh codes like Arepo address this important problem Springel (2011).

\section{Dynamical State: Cold Fronts and Mergers}

Probing cluster emission at larger scales is also helping to illuminate the dynamical response of clusters to recent mergers. While Chandra detected a surprising number of "cold front" contact discontinuities in clusters, a larger field of view of the cluster allows a much more robust identification of the underlying dynamical origin of prominent cold fronts.

The current understanding is that sloshing in response to gravitational perturbations of the cluster center is the primary mechanism for the formation of cold fronts Markevitch et al. (2001), while ram pressure stripping in direct cluster mergers with un-even mass ratios can also lead to cold fronts in some cases Vikhlinin et al. (2001).

Both zoomed and taylored hydrodynamic cluster simulations show that this process works (ZuHone et al. 2013). Recent simulations show that sloshing often leads to the development of characteristic sprial density waves. Emission measure maps of the density distribution from simulations of this effect show a clear set of alternating surface brightness peaks.

Such off-set brightness peaks were recently confirmed by deep XMM imaging of the Perseus cluster Simionescu et al. (2011), but had, in fact, already been seen in Rosat HRI images Churazov et al. (2000). Abell 496 also shows the tell-tale signature of merger induced surface brightness peaks Roediger et al. (2011, 2012). Like tidal tails in galaxy merger studies, these features have the potential to illuminate the merger history of clusters, and provide, in my view, a very promising avenue for detailed cluster studies.

A second important set of recent studies on the observational signatures of dynamical interactions show that radio relics can be produced in merger shocks and trace the merger morphology well. For example, in the case of Abell 3667 Rottgering et al. (1997), the original shock-induced cold front, a set of radio relics is aligned with the merger axis and presumably indicates the current location of the shock. Other clusters show similarly aligned relics, and simulations match the observed relic morphologies well (Hoeft et al. 2011; Skillman et al. 2012). 


\section{Baryon Physics and Dark Matter Profiles}

A third important thread discussed during the cluster theory session is the effect that baryon physics can have on the dark matter profiles in clusters.

While collisionless dark matter is typically assumed to evolve only under the influence of its own self gravity, and that dark-matter-only simulations represent the dark matter profiles resulting from the hierarchical assembly of a halo well, a number of recent studies suggest that gas physics might be more important in the final dark matter profile of a halo than previously assumed.

In the case of galaxy sized halos, it was recently argued that the inclusion of stellar feedback significantly flattens the centeral portion of not just the gas mass profile, but the dark matter profile as well into a cored profile Governato et al. (2012).

Martizzi et al. (2012) presented recent cluster simulations that include AGN feedback, in the form of thermal feedback similar to the supernova feedback used in galactic scale simulations, and found that cluster gas and dark matter mass profiles are affected, reducing the central dark matter densities by about an order of magnitude.

Given that the underlying dark matter physics is scale free, and that comparable feedback prescriptions were employed, it is, perhaps, not surprising that both approaches yield similar results. It does, however, highlight the importance of baryon physics for the dynamical evolution of not just the gas but even the dark matter. Larger samples of simulations that explore these effects will be required to fully understand the effects at play.

\section{Cooling flows/AGN feedback - Observation}

\section{Session chair and section author: Jeremy Lim}

In the classical picture where X-ray-emitting gas comprising the intragroup medium (IGM) or intracluster medium (ICM) cools and flows inwards ("X-ray cooling flow") without being subjected to any external energy injection ("reheating"), the predicted massdeposition rates from such X-ray cooling flows can reach values in excess of $1000 \mathrm{M}_{\odot} \mathrm{yr}^{-1}$ (Fig. 1, left panel). Today, we know that X-ray cooling flows must be strongly mitigated, if not entirely quenched, in all the galaxy groups and clusters so far observed. The most direct evidence comes from the lack of detectable X-ray gas in the cores of galaxy groups or clusters at temperatures below about one-third the bulk ambient temperature, implying that any X-ray cooling flows must have mass-deposition rates at least one-tenth smaller than the classically predicted value. (Alternatively, the X-ray gas penetrates into and mixes with the optical line-emitting gas, so that, effectively, the gas does not cool through X-ray temperatures; e.g., Fabian's talk.) Additional evidence comes from the smaller-than-predicted levels of gas below X-ray temperatures, and rate of star formation ( $\sim 1 \%$ of the classically predicted mass-deposition rate; e.g., McNamara's talk, presented by Nulsen), in the central dominant elliptical galaxies of groups and clusters that, based on the strong X-ray radiative loss at their cores, ought to to harbor X-ray cooling flows.

Jets of relativistic particles (most easily detectable by their synchrotron emission at radio wavelengths) from the central supermassive black hole in the central dominant group or cluster elliptical galaxy have for some time now been invoked to reheat the cooling X-ray gas. The mechanical energy contained in such jets, as inferred from their synchrotron emission (Leith's talk), has long been known to be much more than sufficient 

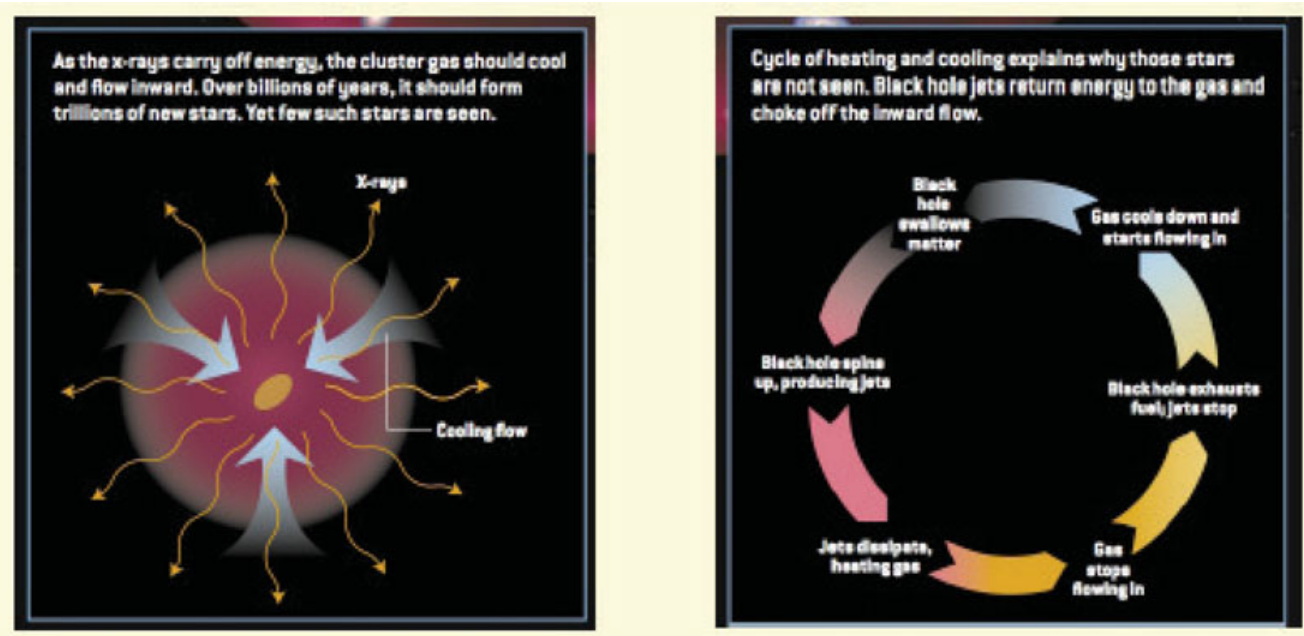

Figure 1. Left panel - X-ray cooling flow in the absence of reheating. Right panel - Feedback between X-ray cooling flow and AGN reheating.

to quench X-ray cooling flows. The primary issues that remain incompletely resolved some would argue entirely unresolved - are:

- How do the jets actually inject energy into the X-ray gas?

- Given that jets are highly collimated, how do they inject energy more or less isotropically into the X-ray gas?

- Why is there an apparent fine tuning such that the inferred AGN heating-rate is approximately equal to the classically predicted X-ray cooling-flow rate?

- Do the jets completely quench the X-ray cooling flow, or is there a residual but still significant X-ray cooling flow?

- If there is a significant residual cooling flow, what is the nature of the cooled gas? Is this gas involved in star formation or fueling of the AGN?

Early ideas that jets inject energy into the IGM or ICM through shocks, and which invoke jet precession to deliver this energy more-or-less isotropically throughout group/cluster cores, have largely — but by no means entirely (e.g., Worrall's talk) — given way to a more nuanced view of reheating thanks to detailed X-ray and radio observations of galaxy groups and clusters (e.g., McNamara's talk and O'Sullivan's talk). X-ray observations reveal cavities in the IGM/ICM in the vicinity of the central dominant elliptical galaxies of groups/clusters expected to harbor X-ray cooling flows; radio observations, in particular those at relatively low frequencies (below $1 \mathrm{GHz}$ ) that probe lower-energy but longerlived synchrotron-emitting electrons, reveal relativistic electrons filling the X-ray cavities, implying that radio jets from the AGN in the central dominant group/cluster elliptical galaxy are responsible for inflating these cavities (Figs. 2-3). The mechanical energy required to inflate the observed cavities (henceforth referred to as the AGN heating-rate) is comparable with the $\mathrm{X}$-ray radiative loss in group/cluster cores, and can therefore strongly mitigate if not entirely quench their X-ray cooling flows. In this manner, jets are able to inject the required energy to counteract cooling over large volumes in the surrounding X-ray gas. The exact manner in which relativistic particles contained in the $\mathrm{X}$-ray cavities transfer their energy to the surrounding X-ray gas, however, remains a subject of debate.

Remarkably, the inferred AGN heating-rate is correlated with the ICM radiative luminosity within the cooling radius (where the cooling time is shorter than the Hubble 


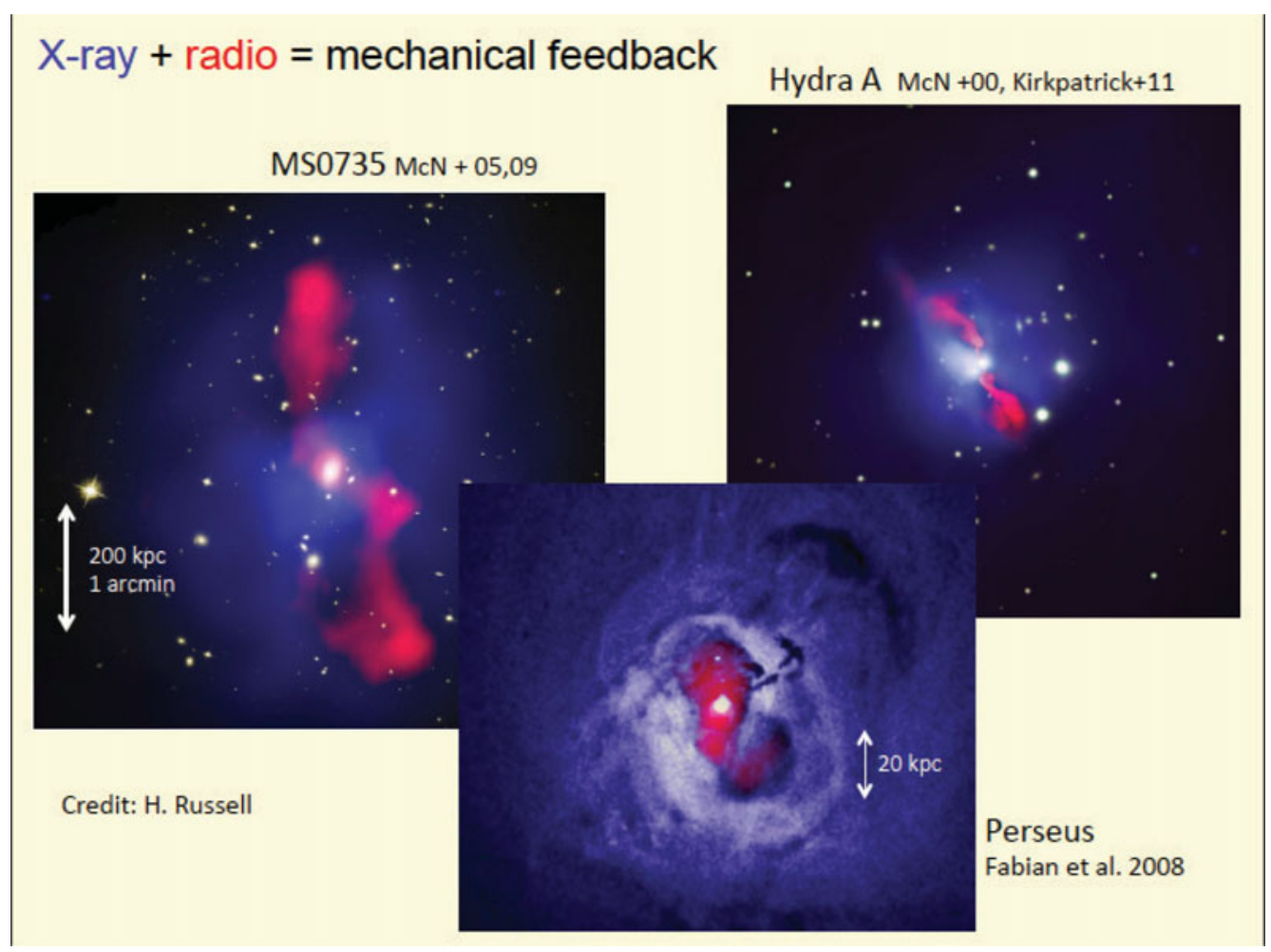

Figure 2. Radio-jet-filled X-ray cavities in galaxy clusters. From McNamara's talk.

time) over seven decades in heating rate/radiative luminosity (McNamara's talk) (Fig. 4, left panel). Such a correlation is also seen in galaxy groups, which of course have lower IGM radiative luminosities, despite the fact that jets often penetrate far beyond the cooling radius in galaxy groups because of their much lower IGM gas pressures; in galaxy groups, less energy contained within the cavity is required to be transferred to entirely reheat the surrounding X-ray gas (O'Sullivan's talk) (Fig. 4, right panel). How does the AGN know about cooling of the ICM given that fueling of the AGN occurs on a spatial scale that is imperceptibly small compared with the volume of the cooling ICM? There must presumably be a feedback process at work (e.g., as proposed in Fig. 1, right panel), but how does this feedback process operate in such a way as to be fined tuned over a tremendously large range in spatial scales and therefore dynamical timescales? Or are we simply witnessing the cumulative effects of repeated episodes of reheating that, on average, produces an apparent relationship between the AGN heating-rate in a given episode (that is comparable to the AGN heating-rate in any given episode) and the timeaveraged response of the IGM/ICM? A basic understanding of this feedback process, of vital importance for informing theoretical simulations on the growth and evolution of massive galaxies, is one of the most difficult yet urgent challenges we now face.

Are X-ray cooling flows completely quenched? Galaxy clusters that ought to harbor strong X-ray cooling flows in the absence of reheating have long been known preferentially to exhibit luminous optical line-emitting gas closely associated with their central elliptical galaxies, and this optical line-emitting gas is usually spatially coincident with the coolest $\mathrm{X}$-ray gas component. This situation is true also in galaxy groups (O'Sullivan's talk). The inferred mass of the optical line-emitting gas, however, is relatively small $\left(\lesssim 10^{7} \mathrm{M}_{\odot}\right)$. On the other hand, over the past decade, surveys in $\mathrm{CO}$ at millimeter wavelengths have 


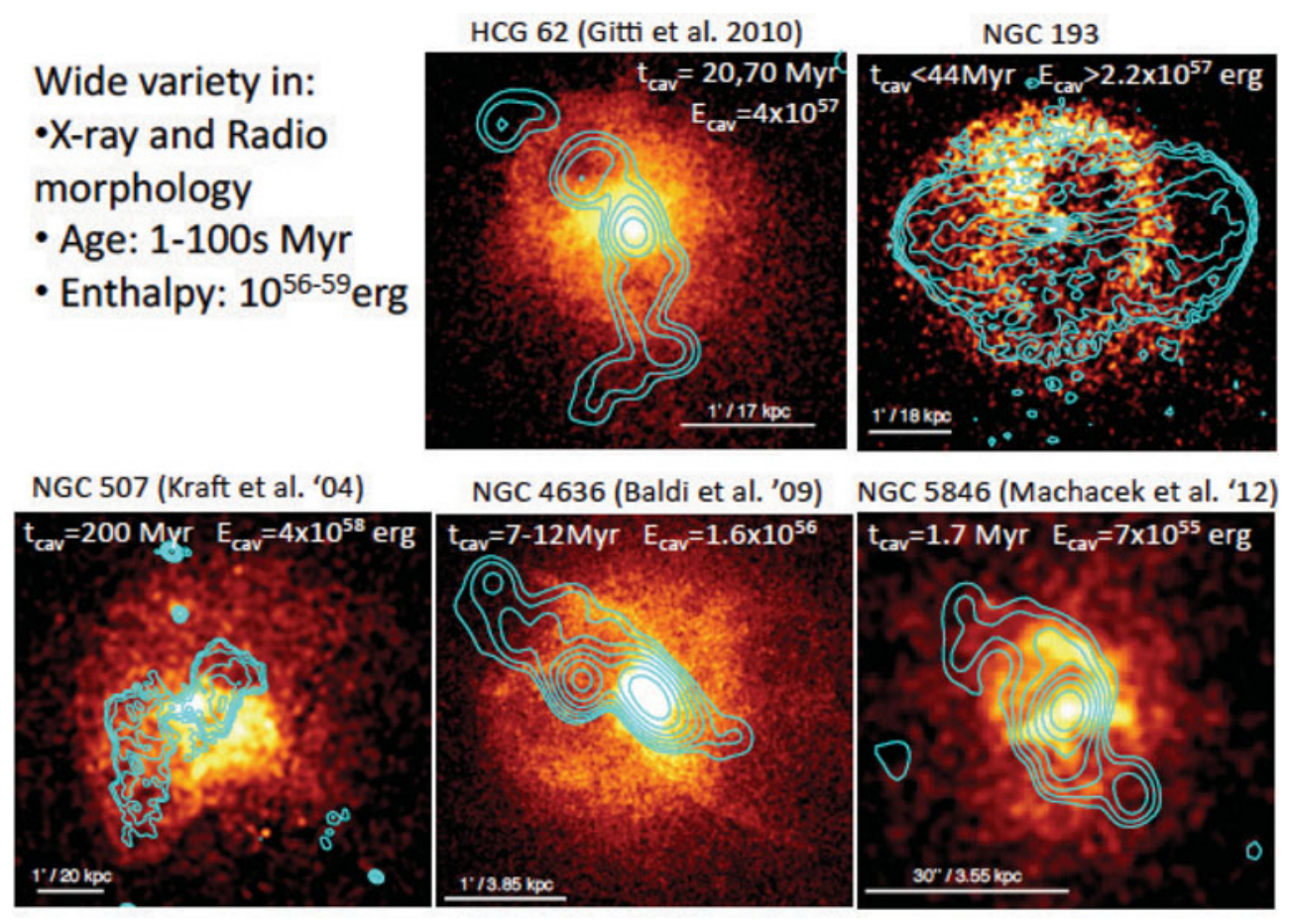

Figure 3. Radio-filled X-ray cavities in galaxy groups. From O'Sullivan's talk.
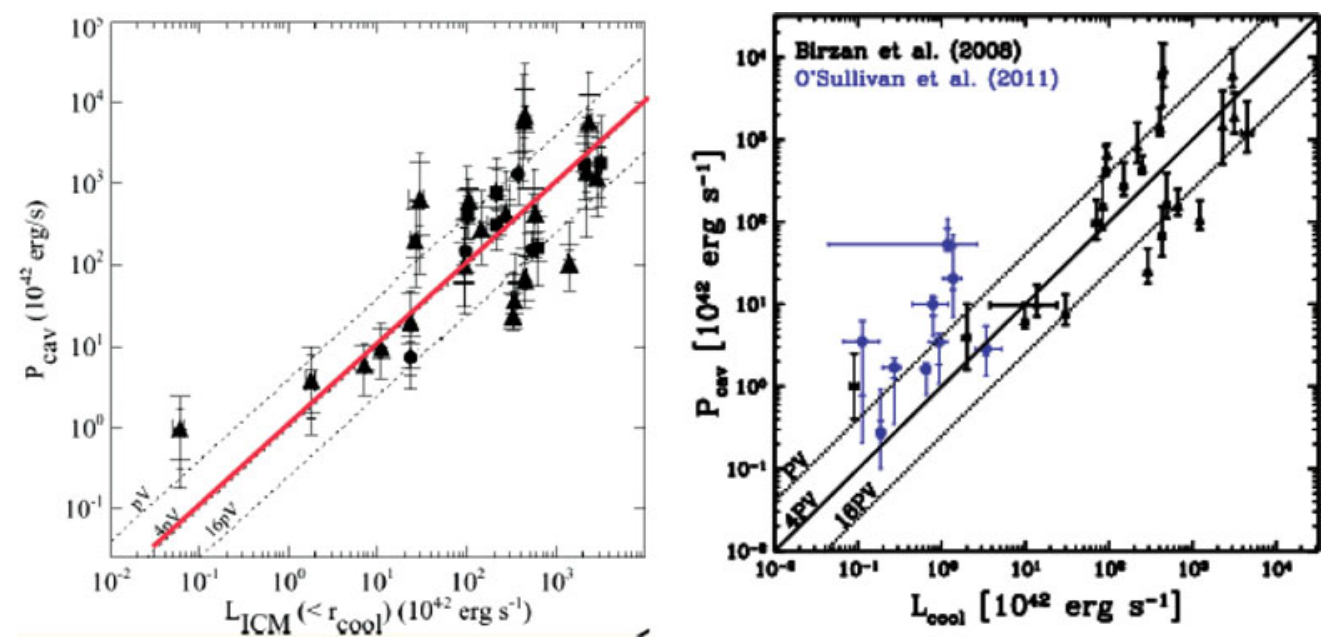

Figure 4. Left panel - Correlation between ICM luminosity within the cooling radius and the mechanical power required to inflate X-ray cavities in clusters. From McNamara's talk. Right panel — Same as left panel, but including galaxy groups. From O'Sullivan's talk.

revealed large masses of cool molecular gas (up to $\sim 10^{11} \mathrm{M}_{\odot}$ ) in the central elliptical galaxies of the same clusters. Is all or a portion of this gas deposited by X-ray cooling flows, and if so was this gas deposited in the distant past or still forming from a residual X-ray cooling flow at the present time? In the core of the Perseus cluster, both atomic and molecular gas are distributed in filaments that are mostly aligned approximately radially although there also are tangential as well as curved filaments. At least some, 
and perhaps all, of these filaments are believed to comprise gas either dragged out by rising X-ray bubbles (i.e., jet-blown X-ray cavities that have become buoyant) or gas draining back into the galaxy after being dragged out by rising bubbles (Chan's talk). In this picture, much if not all of the cool molecular gas, which comprises the bulk of the gas detectable below X-ray temperatures, may have been deposited in the distant past. Alternatively, a portion of the cool molecular gas may have condensed from X-ray gas compressed at the outer surface of rising bubbles, or more simply from a residual X-ray cooling flow, in the recent past. Progress on this front will require studies of the physical properties and relationship between the individual components of the multiphase gas, as well as their spatial relationship with X-ray cavities and bubbles.

Indirect evidence that buoyant X-ray bubbles drag gas from the central elliptical galaxy out to relatively large radii comes from observations of enhanced metallicities along the axis of radio jets (McNamara's talk and O'Sullivan's talk) (Fig. 5), and that the radius over which metal enrichment is observed in galaxy clusters is correlated with the jet mechanical power (McNamara's talk) (Fig. 6). In Abell 2597, relatively cool X-ray gas is seen extending far beyond the north-eastern jet associated with the central cluster elliptical galaxy, suggesting that even cool X-ray gas from the cluster core can be dragged out to relatively large radii by rising X-ray bubbles (Tremblay's talk). Such observations present an alternative picture, or at least an additional pathway, for how the IGM or ICM can be enriched in metals other than through galactic outflows driven by stellar winds or supernova explosions.

\title{
7. Cooling flows/AGN feedback - Theory
}

\author{
Session chair and section author: Daisuke Nagai
}

Introduction: Recent Chandra and XMM-Newton X-ray observations reveal direct evidence of gas heating in the central regions of galaxy clusters, through the interaction of jets and bubbles produced by active galactic nuclei (AGN) with the surrounding intracluster medium (ICM) (Fabian 1994; Peterson \& Fabian 2006; McNamara \& Nulsen 2007). While it is widely believed that AGN can produce enough energy to heat the core, the mechanism of how the energy of the AGN jets and bubbles are transported to the ICM remains unclear.

Numerical simulations of AGN jets and bubbles in galaxy clusters have been performed by a number of authors to investigate this issue. The simulations performed to date are broadly classified into two categories: (i) idealized simulation of 3D spherically symmetric clusters (Quilis et al. 2001; Brüggen \& Kaiser 2002; Omma et al. 2004; Sijacki \& Springel 2006), and (ii) cosmological simulations with sub-grid models of AGN accretion and energy injection (Sijacki et al. 2007; Booth \& Schaye 2009; Teyssier et al. 2011; Yang et al. 2012). While many of the AGN feedback models have been studied using pure hydrodynamic simulations, some of the recent work has investigated the roles of nonideal and/or non-thermal phenomena on the AGN-ICM interactions, including viscosity (Ruszkowski et al. 2004; Reynolds et al. 2005; Sijacki \& Springel 2006), magnetic fields (Dubois et al. 2009) and cosmic-rays (Sijacki et al. 2008), finding that these effects play important roles in modeling the AGN-ICM interactions, interpreting radio and X-ray observations of non-thermal phenomena in clusters, as well as constraining cosmological parameters using X-ray and Sunyaev-Zeldovich (SZ) cluster surveys.

In SpS2 session 6, we discussed recent progress in theoretical modeling of AGN feedback in galaxy clusters. We briefly summarize some of the key findings reported in this section. 
Hydra A Cool, Metal Enriched Outflow

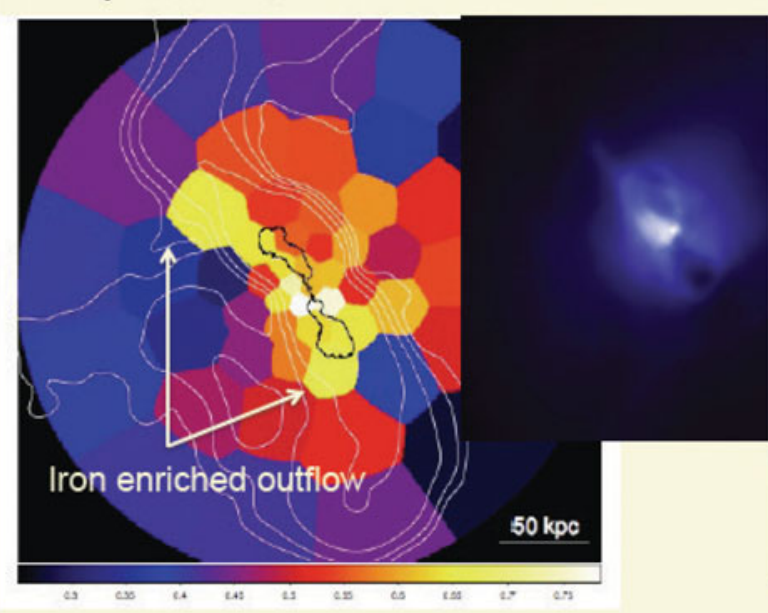

Kirkpatrick +09

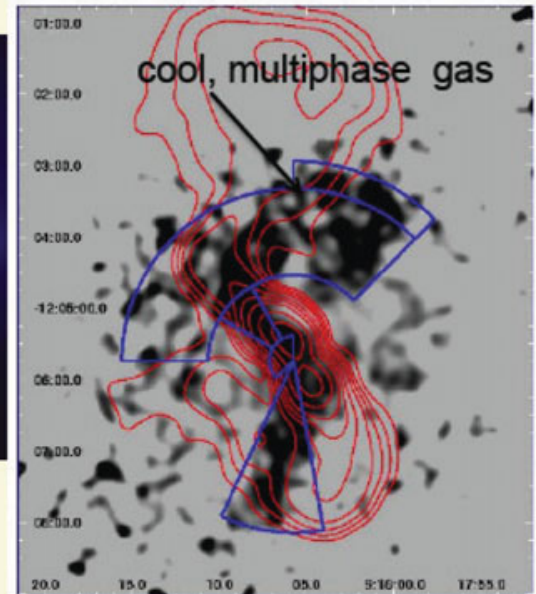

Gitti + 11

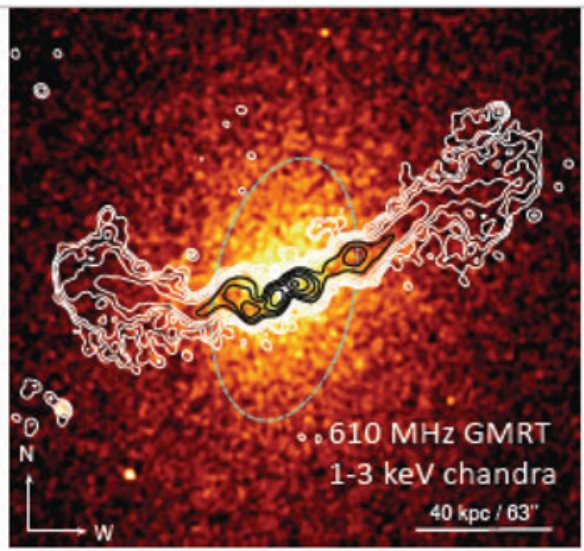

- $10^{9} \mathrm{M}_{\odot}$ gas entrained

- Uplift requires $1.6 \times 10^{57} \mathrm{erg}$, $\sim 5 \%$ of total jet energy.

- $2.5 \mathrm{keV}$ poor cluster, BCG hosts old ( $170 \mathrm{Myr}$ ) active FR-I radio source. - Super-solar abundances along axis of radio jets.

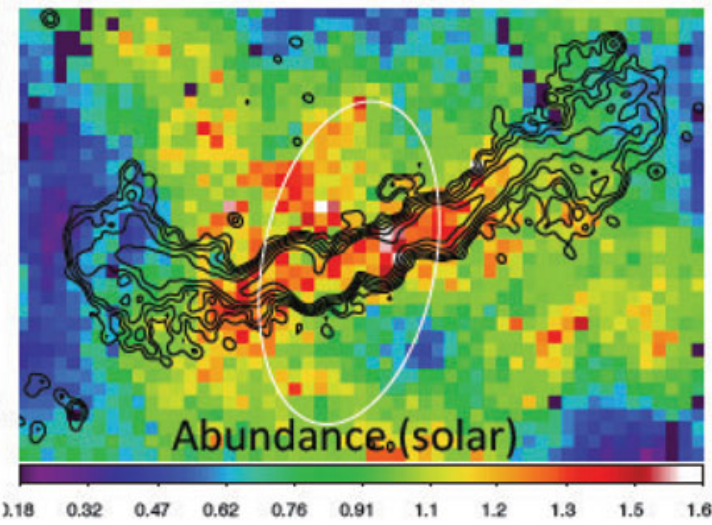

Figure 5. Upper panels - Metal enrichment along the axis of the radio jet from the central elliptical galaxy of a cluster. From McNamara's talk. Lower panel - Metal enrichment along the axis of the radio jet from the central elliptical galaxy of a group. From O'Sullivan's talk.

Simulating the Interaction of Jets with Dynamic Cluster Atmospheres: Heinz et al. presented the FLASH hydrodynamical simulations of the AGN-ICM interaction in realistic, cosmologically evolved cluster atmospheres, focusing on modes of energy transfer from non-thermal to thermal gas and the development of diagnostics one can use to connect simulations to observations (Heinz et al. 2006; Brüggen et al. 2007). Their simulations highlighted that the dynamic state of the cluster is critical for the development of this interaction; i.e., the internal cluster dynamics, excited by the action of the jets themselves, residual motions from previous dynamical encounters, and turbulence 


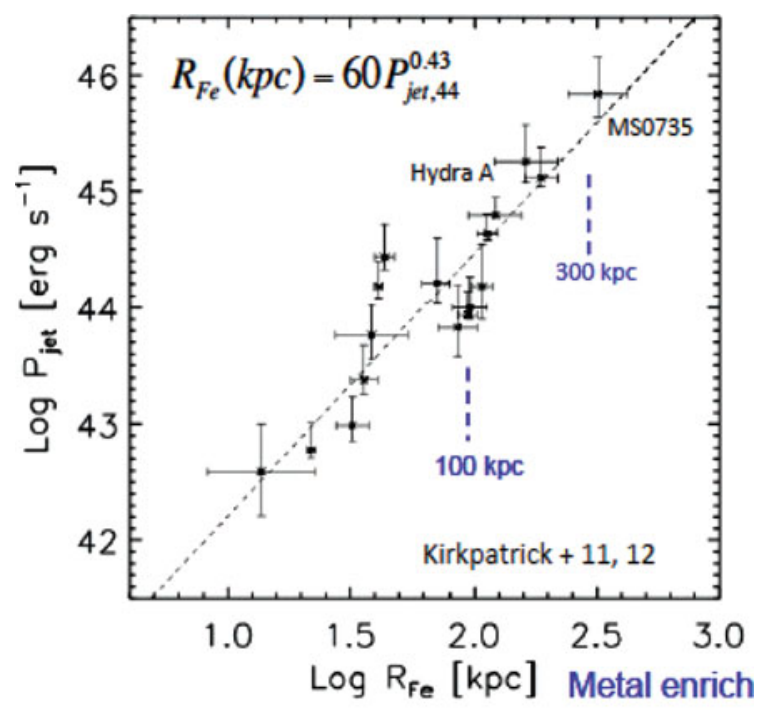

Figure 6. Correlation between metal enrichment radius and inferred jet mechanical power.

From McNamara's talk.

induced by cluster substructure (e.g., moving galaxies), can redistribute the gas in the cluster center in such a way that it erases any channels that were previously carved by the jet. This means that subsequent powerful jet outbursts can efficiently couple with the dense gas of the inner cluster, making it possible for jets to heat cold gas near the centers of clusters. Simulated radio and X-ray maps bear a striking resemblance to observations of prototypical sources, indicating that we are approaching a level of accuracy where detailed re-simulations of individual sources will be possible.

Simulating the AGN Feedback of Cool-Core Clusters: Li \& Bryan carried out high-resolution Enzo AMR simulations of an isolated cool core cluster with and without an AGN jet. The cluster is modeled as a 3D spherically symmetric NFW dark matter halo with gas, BCG, and SMBH, where the initial gas density and temperature is set to reproduce that of the Perseus Cluster, while resolving the flow from Mpc scales down to pc scales (Li \& Bryan 2012). Following Omma et al. (2004), the AGN jet is modeled as the addition of mass, momentum, and energy to cells within the $\mathrm{x}-\mathrm{y}$ plane of the jet launching region, (defined as a cylinder with $r=100 \mathrm{pc}$ and $h=300 \mathrm{pc}$ ), with the total jet power $\left(d E / d t=2 \times 10^{44} \mathrm{erg} / \mathrm{s}\right)$, mass injection rate $\left(d M / d t=6 M_{\odot} / \mathrm{yr}\right)$, kinetic fraction $(f=0.1-1)$, jet speed $\left(v_{\text {jet }}=10^{4} \mathrm{~km} / \mathrm{s}\right.$ for $\left.\mathrm{f}=1\right)$, and precessing jet period $(P=5 \mathrm{Myr})$.

In their pure "cooling-flow" simulations (without AGN), they find that the model cluster develops a global cooling catastrophe and forms a thick accretion disk at the center inside a transition radius of about $50 \mathrm{pc}$ from the SMBH, while local thermal instabilities do not grow outside of this region. When the AGN jet is turned on, their simulations formed cold filaments extending out to $\sim 10 \mathrm{kpc}$, mostly along jet directions. There is a distinct lack of gas below a few $\mathrm{keV}$ in simulations with and without AGN.

Magnetized outflows from AGN in galaxy clusters: Sutter et al. (2012) presented 3D adaptive mesh refinement (AMR) magnetodydrodynamic (MHD) simulations of an isolated galaxy cluster that included injection of kinetic, thermal, and magnetic energy via a central AGN in order to study and evaluate the role that AGN might play in producing the observed cluster-wide magnetic fields. Using the MHD solver in the FLASH 
N-body+hydrodynamic code, they compared several sub-grid models of the evolution of AGN, focusing especially on large-scale AGN jets and bubbles, and examined the effects of magnetized outflows on the accretion history of the black hole and cluster thermodynamic properties. Their study showed that magnetized jet models cause significant reduction in $\mathrm{BH}$ accretion rate compared to hydrodynamic jet models, while the accretion rates in the bubble models remain largely unaffected by the introduction of magnetic fields. Both jet and sporadically-placed bubble models have difficulty reproducing the observed strength and topology of cluster magnetic fields. While the bubble models are generally very unstable, the jet models seem successful in producing weakly magnetized entire clusters and strongly magnetized cluster cores.

Stable Heating of Cluster Cool Cores by Cosmic-Ray Streaming: Fujita \& Ohira (2011) discussed heating of cool cores in galaxy clusters by cosmic-ray (CR) streaming using numerical simulations. In this model, CRs are injected by the central AGN and move outward with Alfvén waves. In this mechanism, CR streaming in the ICM excites Alfvén waves. The CRs interact and move outwards with the waves. The PdV work done by the CRs effectively heats the ICM. Using their simulations, the authors showed that CR streaming can stably heat both high and low temperature clusters for a long time without the assistance of thermal conduction, and it can prevent the development of massive cooling flows. The reason for the stability is that CR pressure is insensitive to changes in the ICM and that the density dependence of the heating term is similar to that of radiative cooling. Moreover, CRs created by the central AGN can stream into larger regions, providing a stable heat source for the entire cluster core. Fujita \& Ohira (2012) also showed that the observed radial profiles of radio mini-halos are remarkably consistent with the predictions of their model.

Impact of AGN Feedback on the Observable-Mass Relations of Galaxy Clusters: Paul Ricker presented Flash AMR simulations of galaxy clusters in both cosmological and isolated contexts to address the influence of feedback from AGN on scatter in cluster X-ray and SZ observable-mass relations (Yang et al. 2012). By performing a detailed parameter sensitivity study in a single cluster using several commonly-adopted AGN accretion and feedback models with FLASH, they quantified the model uncertainties in predictions of cluster integrated properties, including the normalization, slope, and scatter. The study showed that quantities that are more sensitive to gas density have larger uncertainties ( $\sim 20 \%$ for $M_{\text {gas }}$ and a factor of 2 for $L_{X}$ at $R_{500}$ ), while $T_{X}, Y_{\mathrm{sz}}$, and $Y_{X}$ are more robust $\left(\sim 10-20 \%\right.$ at $\left.r=R_{500}\right)$. By studying the impact of AGN feedback on the scaling relations, they found that an anti-correlation exists between $M_{\text {gas }}$ and $T_{X}$, which is another reason why $Y_{S Z}$ and $Y_{X}$ are excellent mass proxies. This anti-correlation also implies that AGN feedback is likely to be an important source of intrinsic scatter in the $M_{\mathrm{gas}}-T_{X}$ and $L_{X}-T_{X}$ relations. Using a sensitivity analysis of subgrid model parameters, they identify mechanical heating efficiency and the size of the feedback region as parameters which, if better constrained by observations, could have the largest impact on model uncertainties in the scatter in the mass-observable relations.

\section{Cold gas and star formation in CFs}

\section{Session chair and section author: Jan Vrtilek}

The results of strong central cooling in cluster gas - the establishment of a powerful cooling flow and the consequent accumulation of large amounts of cold gas at massive central ellipticals - have long been known (e.g., Fabian 1994), as has the absence of 
the expected large amount of star formation. The "problem" was further illuminated by the absence of predicted X-ray spectral cooling features that should have been seen by the sensitive XMM-Newton RGS. The resolution, as discussed in the previous session, is now widely thought to be found in AGN feedback, even if numerous details remain to be understood. Nonetheless, some gas does cool, and the corresponding evidence is to be found in reduced levels of star formation, $\mathrm{H} \alpha$ filaments, and detections of molecular gas. The focus of this session was on the observation, origin, physical state, and fate of that cold material in cluster cores.

It has since the late 1980s been known that it is principally in cool core clusters that cold material — whether atomic or molecular — is to be found. Jeremy Lim reviewed the history of the investigation of this subject, including the correlations between atomic material (as seen in $\mathrm{H} \alpha$ ) and molecular material (detected in CO rotational lines). The detection of even the strongest molecular lines (i.e., low-lying rotational lines of CO) in nearby clusters has not been easy, and surveys have been confined to single-dish detections which, while valuable for multiple purposes (including as pathfinders for future interferometry), do not fully exploit the strength of imaging spectroscopy that radio spectral line work can bring to the subject. Such imaging spectroscopy offers for molecular gas what X-ray spectroscopy cannot do with the current generation of instruments for hot gas: a spatial map of velocities, and the subsequent insight into the movement of material at the centers of cooling flows. To this point interferometry, with PdB or the SMA, has required substantial integration time, even on the strongest and most nearby sources (e.g., Persus or Abell 1795). The advent of ALMA promises a revolution in our understanding by enabling, with practical integration times, the study of a vastly broader array of targets. The first results are now arriving, both in clusters (see, e.g., recent work by McNamara and collaborators on Abell 1664 and Abell 1835) and in groups (NGC 5044: David et al., in preparation). A complication in the interpretation of these results is the $\mathrm{X}$-factor - the conversion beween observed CO and the total molecular column density, which has been calibrated for the particular circumstances common in our Galaxy, but may be quite different elsewhere.

A number of well-studied nearby clusters - e.g., Perseus, Abell 1795, Centaurus exhibit, most clearly in HST images, long (up to $6 \mathrm{kpc}$ ) and very narrow (only $70 \mathrm{pc}$ ) $\mathrm{H} \alpha$ filaments. In Perseus these have been shown to adjoin star forming regions. Andy Fabian reviewed these observations, and argued that the stars cannot be the excitation source for the filaments, as the stars are dynamically unconnected to the gas and will "fall out" of the gas in $\sim 10^{6}$ years. The origin of the filaments is likely from rising bubbles that drag cold gas upwards. Spectra of these filaments are unlike any seen in Galactic objects: there is, for example, no forbidden line emission, such as from [OIII]. If the source of the excitation were from a hot blackbody, its temperature would have to be $\sim 150,000 \mathrm{~K}$. Excitation by energetic particles is instead proposed: these produce showers of secondary electrons on impact.

Based on HST UV images of the CLASH sample (25 massive, dynamically-relaxed clusters), the ACCEPT archival analysis of clusters, and in keeping with the above themes, Megan Donahue noted that BCGs are in general not "red and dead". For example, CLASH has revealed clusters characterized by familiar phenomenology, only at greater redshift: e.g., RXJ $1532+30$, similar to Perseus but at $z=0.363$. From ACCEPT, a central entropy $K_{0}<30 \mathrm{keVcm}^{-2}$ implies a cool core; lower resolution worsens the limit at higher redshift. When star formation is seen, where does the star-forming gas come from? - $\mathrm{H}_{2}$ lines uncorrelated with IR emission from dust are found in such cases that are much stronger than those detected in star-forming galaxies. Is there a "warm" phase? 
A new approach to the examination of cold matter in cluster cores is now available from Herschel data. Francoise Combes reviewed some of the recent findings, based in part on the observation of a dozen clusters in the course of a Herschel key project (PI: A. Edge). This work has revealed gas-to-dust ratios around 100 and temperatures of a few 10s K, remarkably similar to the Milky Way. This could be a surprise: why is the dust not destroyed by sputtering? Lines of [CII] and [OI] are the major coolants of atomic gas; they tend to be spatially associated with $\mathrm{CO}$ emission. The FIR to $\mathrm{CO}$ ratios are typical of those of star-forming galaxies. In Perseus, the [OI] and [CII] line velocities suggest inflow rather than rotation. The [CII] emission tends to be spatially coincident with $\mathrm{H} \alpha$.

At redshifts greater than 0.5 to 0.75 , cool cores as defined by a cuspy surface brightness distribution become rare, but cooling flows identified by cooling time and low central entropy are about as abundant as at low- $z$. Michael McDonald noted this, and raised the question whether optical line emission might be added as a method of CC/NCC classification, a question supported by the recent discovery that the Phoenix cluster supports an $800 \mathrm{M}_{\text {sun }} \mathrm{yr}^{-1}$ starburst and a large and complex emission-line nebula: SZselected clusters are by no means all highly -disturbed "train wrecks" — the Phoenix cluster is not the only one to show strong central cooling.

Based on slit spectroscopy observations along $\mathrm{H} \alpha$ filaments, Jeffrey Chan discussed the structure of the velocity field in Perseus/NGC 1275, revealing a complex structure occasionally confused by overlapping filaments along the line-of-sight. Some filaments may be show rising outer portions and infalling inner portions. Entrainment behind a rising bubble could be involved in some of the observed phenomena.

\section{Ellipticals and Groups of Galaxies}

\section{Session chair and section author: Larry David}

The motion of galaxies through cluster atmospheres can have dramatic affects on the interstellar medium within galaxies as shown by Kraft, who presented X-ray observations and simulations of infalling ellipticals in the Virgo cluster. Kraft presented preliminary results from a deep Chandra observation of the hot ISM of NGC 4552. NGC 4552 lies $350 \mathrm{kpc}$ from M87 in projection, and is presently falling into the Virgo cluster. X-ray observations have shown that the interstellar gas in NGC 4552 has been been stripped during the infall and produced a $20 \mathrm{kpc}$ long tail of stripped gas behind the galaxy. The temperature in the ram-pressure stripped gas increases from $0.5 \mathrm{keV}$ near the galaxy to $1 \mathrm{keV}$ at the edge of the Chandra FOV (see Fig. 7). Specifically tailored viscous hydrodynamical simulations of the infall and stripping process of NGC 4552 were created. The simulations show that if the flow is viscid, then a long, cool tail is formed, similar to what is observed in NGC 4552. However, if the flow is inviscid, small scale KelvinHelmholtz instabilities efficiently mix the stripped gas with the ambient ICM and no tail is formed. The presence of the tail behind NGC 4552 thus suggests that the viscosity of the Virgo cluster ICM is at least a few percent of the Spitzer value.

The Galaxy and Mass Assembly (GAMA) survey provides an opportunity to search for analogues of the Local Group. Robotham gave a presentation based on the analysis of all GAMA galaxies within a factor of two of the stellar mass of the Milky Way (MW) and found that there is a 11.9 close companion within a projected separation of $70 \mathrm{kpc}$, a radial separation of $400 \mathrm{~km} / \mathrm{s}$, and at least as massive as the Large Magellanic Cloud (LMC). There is only a 3.4 finding two close companions at least as massive as the Small Magellanic Cloud (SMC). Only two analogues of the MW-LMC-SMC system were found in GAMA. One example is shown in Fig. 8. These results suggest that such a 


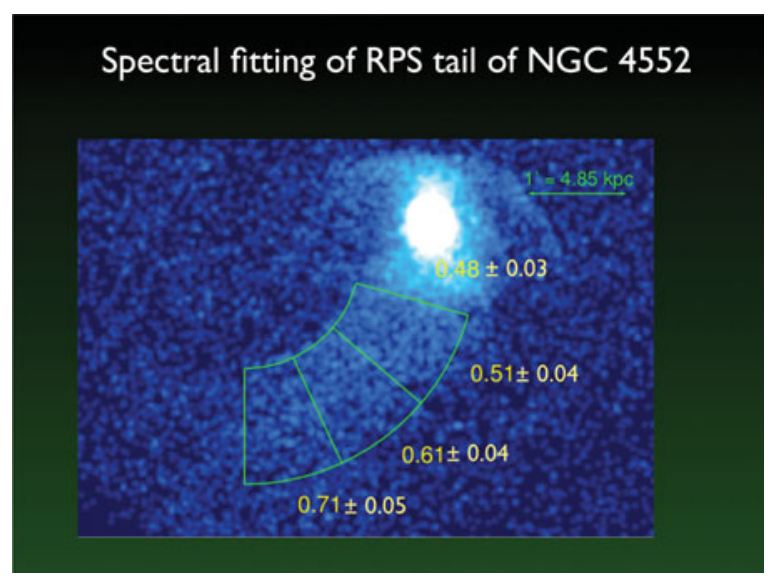

Figure 7. Chandra image of NGC 4552 showing the temperature variation along the tail of ram-pressure stripped gas. (R. Kraft)

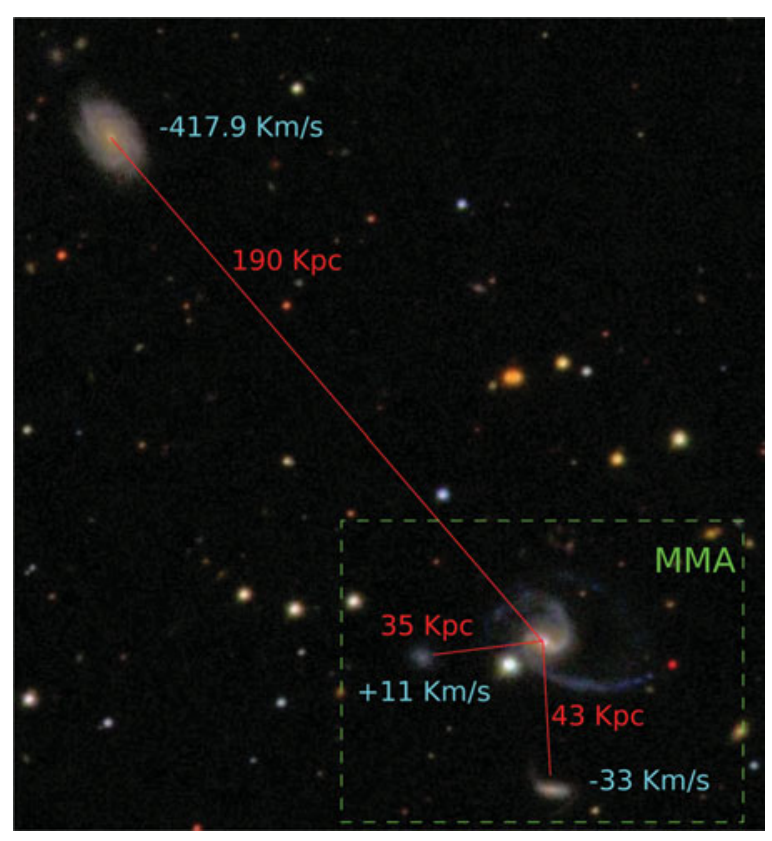

Figure 8. An Analogue to the Local Group

combination of near-by, late-type, star-forming galaxies is rare. Only 0.4 where LMC and SMC mass galaxies could be detected) are embedded in such a system. In summary, the MW-LMC-SMC system is a $2.7 \sigma$ event (when recast into Gaussian statistics).

A question which has been actively debated recently is whether the relationship between halo mass and stellar mass is more fundamental than the relationship between halo mass and velocity dispersion. Li gave a presentation summarizing his work on this question in $\mathrm{Li}$ et al. (2012a) and in $\mathrm{Li}$ et al. (2012b). Li et al. (2012a) recently determined the velocity dispersion profile (VDP) for galaxy groups in SDSS/DR7. They also estimated the redshift-space cross-correlation function (CCF) between central galaxies of a given mass (or luminosity) and a reference galaxy sample. The VDP is then measured by modeling the redshift distortion in the CCF. Within the virial radius, the VDP shows 


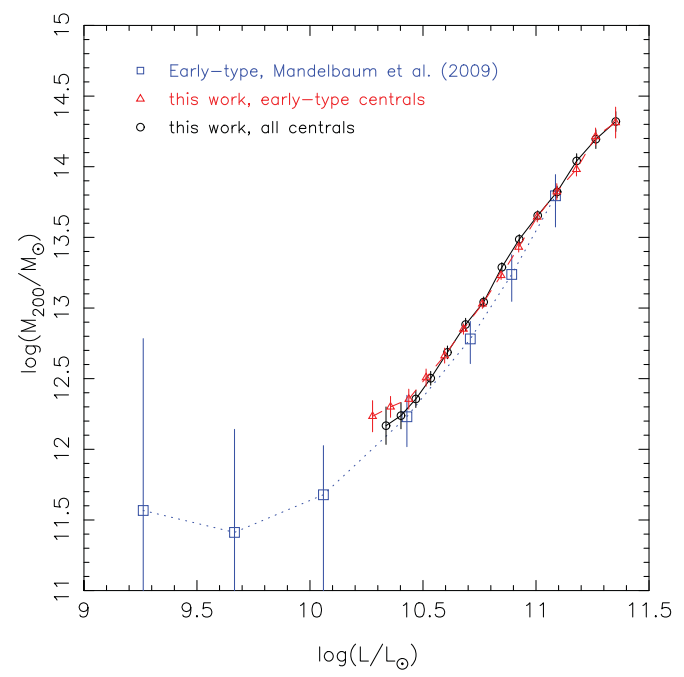

Figure 9. Dark matter halo mass as a function of central galaxy luminosity. Black circles and red triangles are estimated from the velocity dispersion measurements for all central galaxies being studied and for the subset of early-type central galaxies, respectively. Blue squares are the results obtained by Mandelbaum et al. (2006) by stacking the gravitational lensing signals of the early-type central galaxies in the SDSS (Li et al. 2012b)

a roughly flat profile, while the average velocity dispersion (VD) is a strongly increasing function of the central galaxy mass. By applying the same method to N-body cosmological simulations, Li et al. showed that the velocity dispersion vs. stellar mass relation can be used to constrain both the density fluctuation parameter $\left(\sigma_{8}\right)$ and the dispersion in stellar mass at fixed halo mass for central galaxies. Since the velocity dispersion is caused by the local gravitational field, the VDP measurements also provides a direct measure of the dark matter halo mass for central galaxies of different masses and luminosities. As can be seen from Fig. 9, the halo mass vs. luminosity relation agrees well with the results from the weak lensing analysis of Mandelbaum et al. (2006). Li et al. (2012b) extended this work by estimating the halo mass as a function of both the stellar mass $\left(M_{*}\right)$ and stellar velocity dispersion $\left(\sigma_{*}\right)$, and found a much tighter correlation between the halo mass and $M_{*}$ than that between the halo mass and $\sigma_{*}$. This demonstrates that, for central galaxies in halos, the stellar mass is still a good (if not the best) indicator of the host halo mass and is better than the stellar velocity dispersion, which was recently suggested by Wake et al. (2012).

\section{Global radio properties, relativistic particles, and magnetic fields}

\section{Session chair and section author: Diana Worrall}

Although the non-thermal content of clusters makes up only a small fraction of their overall energy, it is key in several areas, such as tracing magnetic field and sites of particle (cosmic ray) acceleration, reflecting merger history, identifying and measuring large-scale outflows from AGN, and indicating AGN life-cycles. This was the subject of Topic 3. It contained a single session with the title "Global Radio Properties, Relativistic Particles, and Magnetic Fields". Two of the invited speakers were unable to attend at the last minute, and while it had been possible to make arrangements for one of the presentations to be given by another speaker, the planned talk from the X-ray perspective was not given. 
Discussion was therefore biased towards what was learned from radio observations, and with a recent growth in radio-astronomy facilities able to map clusters (e.g., GMRT and LOFAR), and upgrades to major facilities such as JVLA and ATCA, there was no shortage of interesting material.

The radio emission of clusters can be grouped into three main types of structures: radio halos, radio relics, and individual active galaxies. They were described in invited talks by Tiziana Venturi and Tracy Clarke (the latter presented by Tiziana Venturi), and Rowan Miller discussed the case study Abell 3266. There were several interesting posters associated with the session, including one by Chernyshov, Dogiel and Ko that discussed the parameter space within which stochastic acceleration of electrons might be responsible for the hard-X-ray excess reported in some clusters.

Radio halo sources cover a large fraction of their host clusters, with overall unpolarized structures that roughly correspond to the morphology mapped in X-ray emitting gas. More than 20 giant halos are known. They are detected in bigger, brighter, hotter, more massive clusters, and correlations have been reported between radio power and X-ray temperature and luminosity.

Radio relic sources have a tendency to lie at the edges of clusters, and are bright where other evidence suggests recent merger activity. About 25 such cases are known. The radio polarization that is seen suggests the presence of strong shocks and the restructuring of magnetic field. Power-law spectra of $\nu^{-\alpha}$ with $\alpha=1.2-1.5$ are typical. The larger relics appear to have flatter spectra related to larger Mach numbers and reside at larger cluster radii. There are examples that are interpreted as adiabatically compressed fossil plasma, and simulations can reproduce the sort of filamentary structure that is seen.

While the existence of radio halos and relics has been known for as long as 50 years, they pose a problem in that the diffusion time across them is typically 100 times longer than the electron energy-loss time. This mandates in-situ electron acceleration. Given that such acceleration must be possible, why are halos and relics a relative rarity? One clear point to emerge is that halos and relics exist only in unrelaxed clusters.

Alternative models have been proposed for particle acceleration in relics and halos. It may arise as a result of strong shocks and MHD turbulence resulting from a cluster merger. Of note are relic structures that are impressively long with very uniform spectral indices. The alternative suggestion is that the electrons are secondaries from charged pion decay after cosmic-ray protons have interacted with thermal protons, although this may disagree with the steep spectrum that is observed, and the accompanying neutral pions would be a source of gamma-rays, in possible contradiction with Fermi results.

A more recent development has been the detection of mini halos (although these can still be of order 100 to $400 \mathrm{kpc}$ in size) in clusters where a central radio galaxy can be the source of relativistic particles. There is an apparent correlation with the presence of cold fronts, often interpreted in the context of gas sloshing, which may provide the source of turbulence for particle acceleration.

Radio galaxies in clusters tend to be distorted in morphology, with more narrow-angletail (NAT) and wide-angle-tail (WAT) examples. Rotation measures of radio galaxies provide estimates of cluster magnetic field strength (typically a few $\mu \mathrm{G}$ ). The enhanced gas density presumably helps to limit adiabatic energy losses, but while the brightest clusters are most likely to host radio galaxies, clusters have little effect on the overall radio-galaxy luminosity function. Abell 3266 is an interesting cluster where there is evidence from spectroscopic redshifts of a merging subcluster and from radio mapping of possible relics. It also hosts a radio galaxy of severely bent NAT morphology that appears to be in-falling from the cluster's edge. Polarization is strongly ordered along the tails, but appears mixed with turbulent field belonging to the cluster itself. 
An obvious conclusion is that clusters are complex beasts, particularly when caught in phases of merging! Deeper radio spectral index and polarization mapping are needed, especially to higher redshift where perhaps NATs and WATs could be used as tracers for clusters. The future is bright for radio mapping, particularly at the low frequencies needed for the steep-spectrum extended structures (e.g., JVLA is replacing the 74 and $330 \mathrm{MHz}$ receivers with improved broad-band capability over 56-470 MHz).

My thoughts from the session are that it might be nice to think there is the potential of discriminating between merging and relaxed clusters through their non-thermal radio content, and that the construction of useful mass scaling relations using the radio might be possible, but all that will depend on findings from deeper radio observations. A better understanding of the locations and mechanisms of particle acceleration is very likely to occur in the near future. A more profound problem is the origin of the magnetic fields.

\title{
11. Galaxy evolution - Part 1 (Clusters)
}

\author{
Session chair and section author: Paulo Lopes
}

Galaxy clusters have been important for galaxy evolution studies in a variety of forms, related to the star formation rate and morphological transformation. In particular, cluster studies based on the colour-magnitude $(\mathrm{CM})$ relation at high-z $(z=1.5)$ show that massive red galaxies with no star formation were fully assembled in mass at $z>2-3$ (Mei et al. 2009). At intermediate to high redshifts $(z<1)$ studies of the evolution in the fraction of blue galaxies indicate the presence of a large population of non-active galaxies. The investigation of the red sequence (RS) faint-to-luminous ratio also points to a progressive assembling of the red sequence for faint objects, indicating that star formation ends in a downsizing way (Stott et al. 2007). The evolution of the morphologydensity relation indicates that a large number of spiral galaxies are transformed into S0s in the last billion years (from $z=1$ to $z=0$; Dressler et al. 1997). A morphological change is also seen in the field, as the early-type fraction grows from high to low-z (Capak et al. 2007).

In the local Universe it has been found that galaxy morphologies and star formation activity do not depend of the parent halo mass (indicated by the cluster velocity dispersion). That is true at least for objects with $\sigma_{c l}>500 \mathrm{~km} \mathrm{~s}^{-1}$ (Poggianti et al. 2009, based on the Wide-Field Nearby Galaxy-Cluster Survey, WINGS). Also based on the WINGS, Valentinuzzi et al. 2011 showed that a number of parameters from the red sequence (RS slope and scatter, red luminous-to-faint fraction, blue fraction, and fractions of ellipticals, S0s, and spirals) correlates with different tracers of cluster mass or substructure indicators (such as velocity dispersion, X-ray luminosity, number of cluster substructures, BCG prevalence and spatial concentration of ellipticals). On the contrary, these authors found that all the above parameters correlate with local density, in agreement with Peng et al. 2012 .

These works suggests that not only galaxy mass, but also local density is a key parameter driving galaxy evolution. The dependence on local density of the galaxy stellar mass function (Vulcani et al. 2012) and of the morphology-mass relation (Calvi et al. 2012) also support that idea (see Figure 1 below). It is well know that morphological transformation happens preferentially on the galaxy group scale (Helsdon \& Ponman 2003; Hoyle et al. 2012). Nonetheless, galaxy clusters still are great places to study galaxy evolution, as cluster specific processes may have a frosting effect on top of the pre-processing on a group level. 


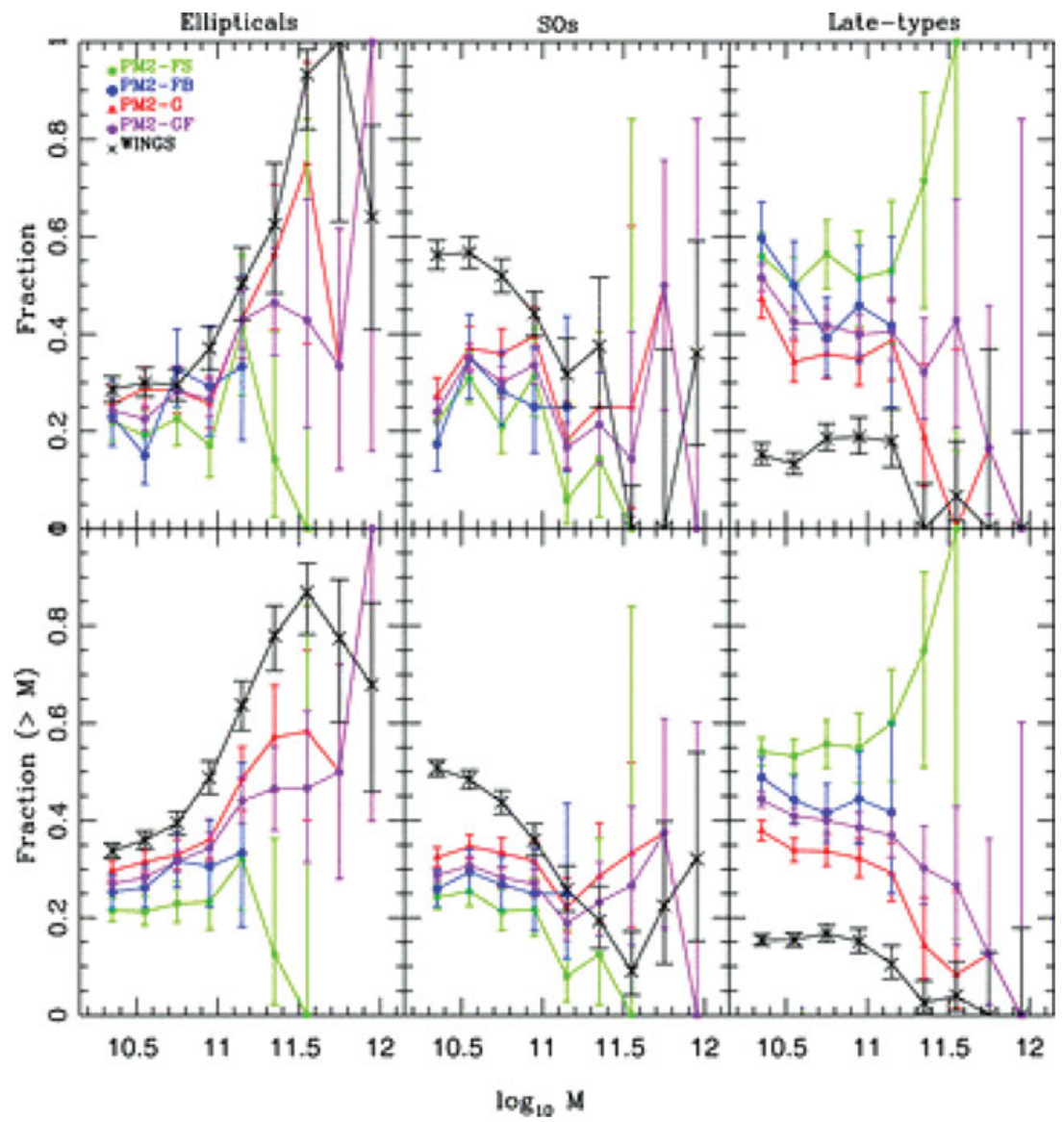

Figure 10. Top panels: fractions of elliptical (left), S0 (central), and late-type (right) galaxies in different environments as a function of stellar mass. Bottom panels: Cumulative distributions of ellipticals, S0s and late-type galaxies. Figure from Calvi et al. 2012.

On what regards high redshift studies it is known that a large fraction of the passive and massive galaxies at $z \sim 2$ end up in clusters at $z=0$. That has implications, for instance, on the evoltuion of galaxy sizes. Investigation of the object Cl J1449+0856 $(z \sim 2)$ show the existence of a concentration of candidate members, classified as early-type galaxies, that are already massive, red, quiescent (Figure 2). Those also are a factor of 2-3 smaller than $z=0$ objects (Strazzullo et al. 2012). On the other hand, the authors find that interlopers at the same redshift $(z \sim 2)$ are more compact than candidate members. The main difficulties on this type of work regards membership determination, as most of the work is based on photometric redshifts. The conclusions above are supported by the work of Strazzullo et al. 2010, based on the cluster XMMU J2235-2557 at $z=1.39$. They find this object already has a tight central red sequence composed of massive early-type galaxies, with negligible star formation.

At $z \sim 1$ Burke et al. 2012, measured for the first time the intra-cluster light (ICL), showing that it represents $1-4 \%$ of the total cluster light. The comparison to nearby objects indicate a strong evolution (growth of 2-4 times) of the ICL component. That is in contrast to the small growth of the BCGs, what suggests that stripping may be dominant over merging in the cluser cores. The authors further sugest that this stripped material should be accounted in cluster evolution simulations. An ongoing study based on 


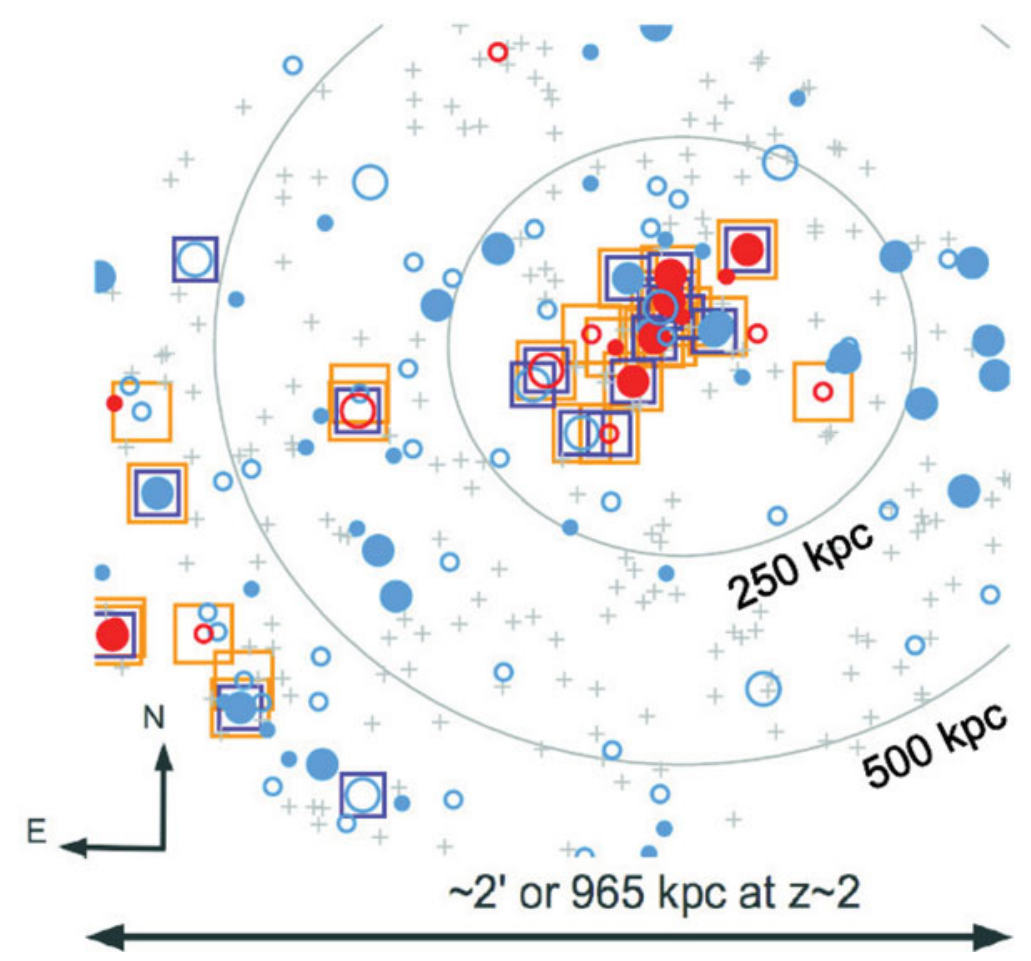

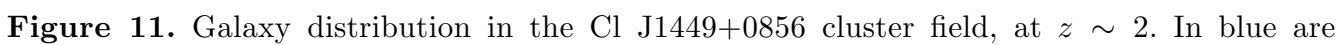
shown star-forming candidate members and in red, quiescent candidate members. Figure from Strazzullo et al. 2012.

IFU observations of galaxies in different local densities shows that in dense environments $\left(\Sigma_{5}>1\right.$ gals $/ \mathrm{Mpc}^{2}$; typical of groups) when the star formation has decreased the gas has been removed from the outskirts. That indicates stripping and/or slow starvation may be acting on these galaxies (Brough et al. 2012).

\section{Galaxy evolution - Part 2 (Groups)}

\section{Session chair: Matthew Colless}

This session focused on the general area of galaxy evolution in groups rather than clusters, and included the following talks: Xiaohu Yang on "Galaxy Evolution in Groups";

Eric Wilcots on "The Assembly and Evolution of Groups of Galaxies";

Marcella Carollo on "Which environment affects galaxy evolution?";

Yingjie Peng on "The quenching of satellite galaxies as the origin of environmental effects";

Simon Lilly on "Galaxy evolution in zCOSMOS groups to z 1"; and Areg Mickaelian on "Multiple galaxies and groups among the BIG objects".

\section{Galaxy evolution - Part 3}

Session chair: Simon Lilly 
This session completed the presentations portion of the meeting with a variety of talks on galaxy groups:

Duncan Forbes on "Galaxy Groups: Results from the GEMS Survey";

Paulo Lopes on "Segregation Effects in Galaxy Groups";

Claudia Mendes de Oliveira on "The Tully-Fisher relation for interacting and field galaxies at $\mathrm{z}=0$ : lessons to be learned for high- redshift TF work";

Mike Hudson on "When, where and how star formation is quenched on cluster infall"; and

Thais Idiart on "Galaxy Evolution through cosmic time".

\section{Concluding panel discussion}

Andy Fabian (chair), Mark Birkinshaw, Marcella Carollo, Ralph Kraft, Daisuke Nagai, and Tiziana Venturi kindly agreed to lead a concluding summary and discussion of open questions to close the meeting.

Among the questions and opportunities for future investigation raised by the panelists and in subsequent discussion with the attendees were the following:

- What is the prevalence of cool cores as a function of redshift? Is this the same in clusters and groups?

- In just a a few years, new low-frequency radio telescopes will begin to have major impact, and will change our view of AGN outbursts. There is much to be done in the comparison of these data and deep targeted X-ray observations.

- The new radio facilities will also open the study of low-mass systems at high redshifts.

- The kind of study that has in the past been applied to massive clusters is moving toward lower masses, with a focus on the statistics of substantial samples and the refinement of physical understanding.

- Two new methods for finding clusters at high redshifts are promising: the SZ effect and the search for radio halos.

- The comparison of optical and X-ray data promises to illuminate group and cluster assembly history.

- The detection of the kinematic SZ effect opens new opportunities.

- N-body and hydrodynamic simulations are already highly valuable, but need to and will improve at fine scales, with the inclusion of better physics. The close connection between observation and simulation is vital.

- The imminent launches of eROSITA and ASTRO-H offer remarkable opportunities for cluster surveys and direct measurements of turbulence in cluster gas, respectively.

- Integral field spectroscopy of large numbers of galaxies is likely to have major impact.

- The combination of surveys using multiple techniques and wavelengths has growing importance.

- Selection effects still plague much of our work; a comprehensive understanding of populations needs increasing attention.

- There are still asymmetries in coverage of the northern and southern skies; these may soon be equalized or reversed, with numerous major southern hemisphere facilities starting up.

- Cluster studies continue to have major impact in cosmology: twenty years ago it was on the determination of $\Omega_{m}$, ten years ago on $\sigma_{8}$ : what will be next?

Your chapter editors would like to conclude with their thanks to the members of the organizing committee, to the session chairs, to the organizers from the Local Committee and from the IAU, and most of all to the many presenters whose research, commit- 
ment, and energetic participation has made this Special Session a memorable summary of current progress in the field and of the outlook for future discovery.

\section{References}

Akamatsu, H., Hoshino, A., Ishisaki, Y., et al. 2011, PASJ

Arnaboldi, M., Ventimiglia, G., Iodice, E., Gerhard, O., \& Coccato, L. 2012, A\&A, 545, A37

Booth, C. M. \& Schaye, J. 2009, MNRAS, 398, 53, 0904.2572

Brough, S., et al. 2012, in preparation

Brüggen, M., Heinz, S., Roediger, E., Ruszkowski, M., \& Simionescu, A. 2007, MNRAS, 380, L67, 0706.1869

Brüggen, M. \& Kaiser, C. R. 2002, Nat, 418, 301, arXiv:astro-ph/0207354

Burke, C., et al. 2012, MNRAS, 425, 2058

Burns, J. O., Skillman, S. W., \& O'Shea, B. W. 2010, ApJ, 721, 1105

Calvi, R., et al. 2012, MNRASL, 419, L14

Capak, P., et al. 2007, ApJS, 172, 284

Chaudhuri, A., Nath, B. B., \& Majumdar, S. 2012, ApJ, 759, 87

Churazov, E., Forman, W., Jones, C., \& Boehringer, H. 2000, A\& A, 356, 788

Dressler, A., et al. 1997, ApJ, 490, 577

Dubois, Y., Devriendt, J., Slyz, A., \& Silk, J. 2009, MNRAS, 399, L49, 0905.3345

Eckert, D., Vazza, F., Ettori, S., et al. 2012, Astronomy \& Astrophysics, 541, A57

Fabian, A. C. 1994, ARA $8 A, 32,277$

Fujita, Y. \& Ohira, Y. 2011, ApJ, 738, 182, 1106.5790

—. 2012, ApJ, 746, 53, 1111.4208

Governato, F., Zolotov, A., Pontzen, A., et al. 2012, MNRAS, 422, 123

Heinz, S., Brüggen, M., Young, A., \& Levesque, E. 2006, MNRAS, 373, L65, arXiv:astro$\mathrm{ph} / 0606664$

Helsdon, S. \& Ponman, T., 2003, MNRAS, 339, 29

Hoeft, M., Nuza, S. E., Gottlöber, S., et al. 2011, Journal of Astrophysics and Astronomy, 32, 509

Hoyle, B., et al. 2012, MNRAS, 423, 3478

Janowiecki, S., Mihos, J. C., Harding, P., et al. 2010, ApJ, 715, 972

Li, Y. \& Bryan, G. L. 2012, ApJ, 747, 26, 1112.2701

Li, C., Jing, Y. P., Mao, S., et al. 2012a, ApJ, 758, 50

Li, C., Wang, L. X., \& Jing, Y. P. 2012, arXiv:1210.5700

Lin, Y.-T., Stanford, S. A., Eisenhardt, P. R. M., et al. 2012, ApJ, 745, L3

Mandelbaum, R., Seljak, U., Kauffmann, G., Hirata, C. M., \& Brinkmann, J. 2006, MNRAS, 368,715

Markevitch, M., Vikhlinin, A., \& Mazzotta, P. 2001, ApJ, 562, L153

Markevitch, M., Gonzalez, A. H., David, L., et al. 2002, ApJ, 567, L27

Martizzi, D., Teyssier, R., Moore, B., \& Wentz, T. 2012, MNRAS, 422, 3081

McNamara, B. \& Nulsen, P. E. J. 2007, ARAA, 45, 117

McNamara, B. R., Kazemzadeh, F., Rafferty, D. A., et al. 2009, ApJ, 698, 594

McNamara, B. R. \& Nulsen, P. E. J. 2007, ARA\&AA, 45, 117, 0709.2152

Mei, S., et al. 2009, ApJ, 690, 42

Mihos, J. C., Harding, P., Feldmeier, J., \& Morrison, H. 2005, ApJ, 631, L41

Miller, E. D., Bautz, M., George, J., et al. 2012, in SUZAKU 2011: Exploring the X-ray Universe: Suzaku and Beyond. AIP Conference Proceedings, Vol. 1427, 13-20

Nagai, D. \& Lau, E. T. 2011, ApJ, 731, L10

Nulsen, P. E. J., McNamara, B. R., Wise, M. W., \& David, L. P. 2005, ApJ, 628, 629

Omma, H., Binney, J., Bryan, G., \& Slyz, A. 2004, MNRAS, 348, 1105, arXiv:astro-ph/0307471

Owers, M. S., Couch, W. J., Nulsen, P. E. J., \& Randall, S. W. 2012, ApJ, 750, L23

Peng, Y., et al. 2012, ApJ, 757, 4

Peterson, J. R. \& Fabian, A. C. 2006, Phys. Rep., 427, 1, arXiv:astro-ph/0512549 
Poggianti, B., et al. 2009, ApJ, 697, 137

Quilis, V., Bower, R. G., \& Balogh, M. L. 2001, MNRAS, 328 , 1091, arXiv:astro-ph/0109022

Reynolds, C. S., McKernan, B., Fabian, A. C., Stone, J. M., \& Vernaleo, J. C. 2005, MNRAS, 357, 242, arXiv:astro-ph/0402632

Roediger, E., Brüggen, M., Simionescu, A., et al. 2011, MNRAS, 413, 2057

Roediger, E., Lovisari, L., Dupke, R., et al. 2012, MNRAS, 420, 3632

Rottgering, H. J. A., Wieringa, M. H., Hunstead, R. W., \& Ekers, R. D. 1997, MNRAS, 290, 577

Ruszkowski, M., Brüggen, M., \& Begelman, M. C. 2004, ApJ, 615, 675, arXiv:astro-ph/0403690

Sanders, J. S., Fabian, A. C., \& Smith, R. K. 2010, MNRAS, 410, no

Shang, C. \& Oh, S. P. 2012, MNRAS, 426, 3435

Sijacki, D., Pfrommer, C., Springel, V., \& Enßlin, T. A. 2008, MNRAS, 387, 1403, 0801.3285

Sijacki, D. \& Springel, V. 2006, MNRAS, 371 , 1025, arXiv:astro-ph/0605301

Sijacki, D., Springel, V., Di Matteo, T., \& Hernquist, L. 2007, MNRAS, 380, 877, 0705.2238

Simionescu, A., Allen, S. W., Mantz, A., et al. 2011, Science, 331, 1576

Simionescu, A., Werner, N., Urban, O., et al. 2012, ApJ, 757, 182

Skillman, S. W., Xu, H., Hallman, E. J., et al. 2012, arXiv:1211.3122

Springel, V. 2011, arXiv:1109.2218

Sutter, P. M., Yang, H.-Y. K., Ricker, P. M., Foreman, G., \& Pugmire, D. 2012, MNRAS, 419, 2293, 1108.3344

Tamura, T., Sekiya, N., Hayashida, K., Ueda, S., \& Nagai, M. 2011, in SUZAKU 2011: Exploring the X-ray Universe: Suzaku and Beyond. AIP Conference Proceedings, Vol. 1427, 332-333

Tamura, T., Hayashida, K., Ueda, S., \& Nagai, M. 2012, PASJ, 1427, 332

Simionescu, A., Allen, S. W., Mantz, A., et al. 2011, Science (New York, N.Y.), 331, 1576

Stott, J., et al. 2007, ApJ, 661, 95

Strazzullo, V., et al. 2010, A\& A, 524, 17

Strazzullo, V., et al. 2012, in preparation

Teyssier, R., Moore, B., Martizzi, D., Dubois, Y., \& Mayer, L. 2011, MNRAS, 414, 195, 1003.4744

Valentinuzzi, T., et al. 2011, A\&SA, 536, 34

van Weeren, R. J., Röttgering, H. J. A., Brüggen, M., \& Hoeft, M. 2010, Science, 330, 347

Vikhlinin, A., Markevitch, M., \& Murray, S. S. 2001, ApJ, 551, 160

Vikhlinin, A. A. \& Markevitch, M. 2002, Astron. Let., 28, 495

Vulcani, B., et al. 2012, MNRAS, 420, 1481

Wake, D. A., Franx, M., \& van Dokkum, P. G. 2012 (arXiv:1201.1913)

Walker, S. A., Fabian, A. C., Sanders, J. S., \& George, M. R. 2012, MNRAS: Letters, 427, 45

Yang, H.-Y. K., Sutter, P. M., \& Ricker, P. M. 2012, ArXiv e-prints, 1207.6106

ZuHone, J. A., Markevitch, M., Ruszkowski, M., \& Lee, D. 2013, ApJ, 762, 69 